\title{
Preoperative Predictors of Early Mortality Risk in Isocitrate Dehydrogenase-Wild-Type Glioblastoma Patients Treated with Standard
} Therapy

This article was published in the following Dove Press journal:

Cancer Management and Research

\author{
Chao Zhaol,* \\ Longqing $\mathrm{Li}^{2, *}$ \\ Xiaoyue Guo' \\ Dixiang Song' \\ Minkai Wang' \\ Yixuan Zhai ${ }^{1}$ \\ Fengdong Yang' \\ Yake Xue \\ Xinting Wei ${ }^{1}$ \\ 'Department of Neurosurgery, The First \\ Affiliated Hospital of Zhengzhou \\ University, Henan, People's Republic of \\ China; ${ }^{2}$ Department of Orthopedic \\ Surgery, The First Affiliated Hospital of \\ Zhengzhou University, Henan, People's \\ Republic of China \\ *These authors contributed equally to \\ this work
}

Correspondence: Xinting Wei Department of Neurosurgery, The First Affiliated Hospital of Zhengzhou University, Zhengzhou, 450052, No. I East Jianshe Road, District of Erqi, Henan, People's Republic of China

Tel +86-037I-I3613860990

Email weixinting777@|26.com
Purpose: Early identification of early mortality for glioblastoma (GBM) patients based on laboratory findings at the time of diagnosis could improve the overall survival. The study aimed to explore preoperative factors associated with higher risk of early death (within 1 year after surgery) for isocitrate dehydrogenase (IDH) -wild-type (wt) GBM patients.

Patients and Methods: We conducted a retrospective analysis of 194 IDH-wt GBM patients who underwent standard treatment. The probability of dying within 1 year after gross total resection (GTR) was defined as the end point "early mortality". Retrospective collection of predictive factors including clinical characteristics and laboratory data at diagnosis.

Results: Median follow-up time after GTR was 16 months (3-41 months). Forty-two patients died within 1 year after surgery (1-year mortality rate: $21.6 \%$ ). All potential predictive factors were assessed on univariate analyses, which revealed the following factors as associated with higher risk of early death: older age $(P=0.013)$, occurrence of nonseizures symptoms $(P=0.042)$, special tumor positions $(\mathrm{P}=0.046)$, higher neutrophil-tolymphocyte ratio (NLR) $(P=0.015)$, higher red blood cell distribution width $(\mathrm{RDW})(P=$ $0.019)$, higher lactate dehydrogenase $(\mathrm{LDH})(P=0.005)$, and higher fibrinogen $(\mathrm{FIB})(\mathrm{P}=$ 0.044). In a multivariate analysis, tumor location $(\mathrm{P}=0.012), \mathrm{NLR}(\mathrm{P}=0.032)$ and LDH $(\mathrm{P}$ $=0.002$ ) were independent predictors of early mortality. The C-index of the nomogram was 0.795 . The calibration curve showed good agreement between prediction by nomogram and actual observation.

Conclusion: Tumor location, preoperative elevated NLR and serum LDH level were independent predictors for 1-year mortality after GTR. We indicate that increased preoperative NLR or LDH may guide patients to review head magnetic resonance imaging (MRI) more frequently and regularly to monitor tumor progression.

Keywords: glioblastoma, early mortality, risk factors, survival

\section{Introduction}

Glioblastoma multiforme (GBM) is the most common and lethal type of cerebral tumor, accounting for $57 \%$ of all gliomas and $48 \%$ of all primary malignant central nervous system tumors, with the age adjusted average annual incidence of 3.19 per 100 000 individuals. $^{1,2}$ The incidence associated with progressive decline in neurological function and quality of life can have a devastating impact on patients and their families. ${ }^{3}$ In 2016, the World Health Organization reclassified the definition of GBM, 
dividing these tumors into 90\% IDH-wt and 10\% IDHmutant GBM. IDH1/2 mutations are rare in GBM and generally carry a better prognosis, while IDH-wt GBM comes to be regarded as a more delineated entity: the most common and malignant astrocytic glioma. ${ }^{4}$ The first stage treatment for newly diagnosed GBM is maximally safe surgical resection, followed by concurrent chemoradiotherapy and adjuvant therapy with temozolomide. Over the years, with advances in surgical and radio-chemotherapeutics treatments, the current patient overall median survival has been improved to $>15$ months. ${ }^{5-7}$ Noteworthy, the 1 -year relative survival rate for patients diagnosed in the United States between 2000 and 2014 was estimated to be only about $41.4 \%{ }^{8}$ Recently, more and more attention has been paid to the inclusion of predictive factors used for appropriate risk classification into new strategies for cancer therapy in order to improve the survival time of patients with GBM.

Early mortality in GBM patients is associated with various factors. Some GBM patients may die in the short term after surgical resection, mainly due to established adverse factors such as poor performance status, combination of other malignancies, incomplete extent of resection, and lack of adjuvant therapy, rather than the tumor's own mechanism of action. ${ }^{9-11}$ In the clinical process, we found that those patients who were in good performance status and received standard treatment could still die unexpectedly within the first year from surgical resection. Therefore, the preoperative identification of those patients is of strong clinical value, as those patients can be timely selected for further radical treatment, clinical trial consideration, and supportive care to improve oncologic outcomes.

Mounting evidence has revealed that preoperative hematological biomarkers, which reflect the tumor microenvironment of the body to a certain extent, could serve as diagnostic and prognostic markers for human cancers. ${ }^{12,13}$ Previous investigations have indicated that markers of inflammatory response, such as NLR, platelet-to-lymphocyte ratio (PLR), and lymphocyte-to-monocyte ratio (LMR), are associated with the clinical outcomes of gliomas, especially GBM. ${ }^{14,15}$ Several studies have indicated that a state of preoperative hypercoagulability is related to poor prognosis in patients with GBM. ${ }^{16,17}$ Other factors such as glucose (GLC), albumin-to-globulin ratio (AGR), the prognostic nutrition index (PNI), LDH and RDW have also been shown to have prognostic value in GBM. ${ }^{18-22}$

To date, little knowledge is available about the early death rate and preoperative factors related to early death in GBM patients treated with standard therapy. The objective of this study was to explore preoperative predictors associated with higher risk of early mortality (within 1 year after GTR) for IDH-wt GBM patients.

\section{Patients and Methods Patients}

The retrospective study included patients who were newly diagnosed with GBM between June 2016 and August 2019 at the First Affiliated Hospital of Zhengzhou University. Patients meeting the following criteria were included: 1) patients with IDH-wt GBM confirmed by histopathology; 2) patients aged $\geq 18$ years; 3) preoperative and postoperative Karnofsky Performance Status (KPS) scores $\geq 70$; 4) patients with complete data of routine blood test, coagulation function and hepatic function results; 5) radiotherapy plus concomitant and adjuvant temozolomide were the Stupp regimen; The exclusion criteria were as follows: 1) patients with a clinical history of more than 1 year; 2) patients with midline GBM; 3) patients with obvious infection, autoimmune or hematological diseases; 4) patients with other malignancies; and 5) perioperative surgery-related mortality. A total of 452 patients with GBM were identified from our database, and 194 patients were finally enrolled. All patients enrolling in the current study underwent standard treatment, including GTR, followed by concurrent chemoradiotherapy and adjuvant therapy with temozolomide. No patients received chemotherapy or radiotherapy before the operation. Each patient was followed up regularly until death or October 2020. The patients were observed once every month in the first 6 months after surgery and then every 3-6 months thereafter. The Ethics Committee of the First Affiliated Hospital of Zhengzhou University approved this study and requirement for signed informed consent from patients was waived because of the retrospective nature of the analysis. All patient data were treated with confidentiality, in accordance with the Declaration of Helsinki.

\section{Data Collection}

The primary objective was the identification of preoperative predictors of early death, which were defined as death within 1 year after GTR. ${ }^{23,24}$ As previous studies, a KPS score $\geq$ 70, which was equivalent to the Eastern Cooperative Oncology Group Performance Status (ECOG PS) score $\geq$ 1 , was considered as the good performance status. ${ }^{25-28}$ The following variables were collected from hospital case documents: age at diagnosis, sex, first presenting symptoms, clinical history, KPS score, tumor size, tumor location, and 
laboratory data. Preoperative laboratory markers included: NLR = neutrophil-to-lymphocyte ratio, PLR = platelet-tolymphocyte ratio, LMR = lymphocyte -to- monocyte ratio, $\mathrm{PNI}=$ the albumin value $(\mathrm{g} / \mathrm{L})+0.005 \times$ peripheral lymphocyte count $/ \mathrm{mm}^{3}, \mathrm{AGR}=$ albumin-to-globulin ratio, $\mathrm{RDW}=$ red blood cell distribution width, GLC $=$ glucose, $\mathrm{LDH}=$ lactate dehydrogenase, FIB = fibrinogen levels, and DD = D-dimer levels. Overall survival (OS) as the endpoint was defined as the interval between tumor resection and death or the last date of follow-up. Clinical history referred to the time from onset of symptoms to hospital admission.

\section{Statistical Analysis}

Differences in continuous variables were analyzed using the independent sample $t$-test while categorical variables were evaluated using Chi-squared test or Fisher's exact test as appropriate. Receiver operating characteristic (ROC) curve analysis was used to determine the optimal cutoff value of each hematological marker according to the area under the ROC curve (AUC). Patients were classified into binary categories according to the cutoff values. Univariable Cox analysis was performed with all patients' clinical covariates to screen out the factors associated with early mortality. All covariates significant on univariate analysis were included in multivariate Cox analysis. The hazard ratio and $P$-value of covariates from the Cox analysis were visualized by forest plot. Survival curves were depicted using the Kaplan-Meier method and compared using the Log rank test. A nomogram used to predict the probability of early death was formulated based on the results of multivariate analysis. The performance of the nomogram was measured by $\mathrm{C}$-index and assessed by comparing nomogram-predicted versus observed Kaplan-Meier estimates of survival probability. Decision curve analysis (DCA) was used to evaluate the clinical application of the nomogram. Statistical analyses were performed using SPSS software (version 21.0), and R software (version 3.3.0). $\mathrm{P}<0.05$ was considered statistically significant.

\section{Results}

\section{Patient Characteristics}

The clinical characteristics of all patients are shown in Table 1. During the follow-up process, the median OS durations after GTR was 16 months (3-41 months). Median patient age was 55 years (range 19 to 80 ), median clinical history was 20 days (range 1 to 365 ), $59.3 \%$ of the patients were men, $36.6 \%$ had tumor size $>5 \mathrm{~cm}$, and $11.4 \%$ had seizures as the first presenting symptom. The
Table I Summary of Clinical Characteristics of GBM Patients Who Received Standard Treatment

\begin{tabular}{|c|c|}
\hline Characteristics & All Patients, $\mathbf{N}=194$ \\
\hline \multicolumn{2}{|l|}{ Age, years } \\
\hline Median & 55 \\
\hline Range & $19-80$ \\
\hline \multicolumn{2}{|l|}{ Clinical history, days } \\
\hline Median & 20 \\
\hline Range & $1-365$ \\
\hline \multicolumn{2}{|l|}{ Sex, n (\%) } \\
\hline Male & $115(59.3)$ \\
\hline Female & $79(40.7)$ \\
\hline \multicolumn{2}{|l|}{ Size, cm, n (\%) } \\
\hline$\leq 5$ & $123(63.4)$ \\
\hline$>5$ & $7 I(36.6)$ \\
\hline \multicolumn{2}{|c|}{ First presenting symptoms, n (\%) } \\
\hline Seizures & $22(I I .4)$ \\
\hline Others & $172(88.6)$ \\
\hline \multicolumn{2}{|l|}{ Tumor location, n (\%) } \\
\hline Frontal & $45(23.2)$ \\
\hline Temporal & $43(22.2)$ \\
\hline Mixed & $69(35.6)$ \\
\hline Others & $37(19.1)$ \\
\hline NLR, median (IQR) & $2.37(1.69-4.19)$ \\
\hline PLR, median (IQR) & I30.04 (93.42-174.22) \\
\hline LMR, median (IQR) & $3.47(2.63-4.73)$ \\
\hline PNI, median (IQR) & $50.0(47.2-53.6)$ \\
\hline AGR, median (IQR) & $1.75(1.53-1.98)$ \\
\hline RDW, \%, median (IQR) & $13.3(12.8-13.9)$ \\
\hline GLC, mmol/L, median (IQR) & $4.97(4.48-5.75)$ \\
\hline LDH, U/L, median (IQR) & $176(157-200)$ \\
\hline FIB, g/L, median (IQR) & $3.01(2.55-3.65)$ \\
\hline DD, mg/L, median (IQR) & $0.11(0.08-0.17)$ \\
\hline \multicolumn{2}{|l|}{ OS, months } \\
\hline Median & 16 \\
\hline Range & $3-41$ \\
\hline \multicolumn{2}{|l|}{ Survival Status, n (\%) } \\
\hline Alive & $60(30.9)$ \\
\hline Dead & $134(69.1)$ \\
\hline
\end{tabular}

Abbreviations: NLR, the neutrophil-to-lymphocyte ratio; PLR, the platelet-tolymphocyte ratio; LMR, the lymphocyte -to- monocyte ratio; PNI, the prognostic nutrition index; AGR, albumin-to-globulin ratio; RDW, red blood cell distribution width; GLC, glucose; LDH, lactate dehydrogenase; FIB, fibrinogen; DD, D-dimer; $\mathrm{IQR}$, interquartile range; OS, overall survival.

top three common tumor locations were the mixed lobe location for 69 (35.6\%), frontal lobe location for 45 
$(23.2 \%)$, and temporal lobe location for $43(22.2 \%)$, respectively. Median NLR: 2.37 (IQR 1.69-4.19), PLR: 130.04 (IQR 93.42-174.22), LMR: 3.47 (IQR 2.63-4.73), PNI: 50.0 (IQR 47.2-53.6), AGR: 1.75 (IQR 1.53-1.98), RDW: 13.3 (IQR 12.8-13.9), GLC: 4.97 (IQR 4.48-5.75), LDH: 176 (IQR 157-200), FIB: 3.01 (IQR 2.55-3.65), DD: 0.11 (IQR 0.08-0.17). In Supplementary Table 1, we determined the cutoff values of ten hematological factors for OS by ROC curve analysis (NLR-1.706, PLR87.857, LMR-3.067, PNI-56.5, AGR-1.908, RDW-12.8, GLC-6.57, LDH-182, FIB-3.81, DD-0.183). Then, patients were classified into low-risk and high-risk groups according to the cutoff points. Table 2 compares the preoperative risk factors of postoperative death within 1 year after GTR. 134 patients (69.1\%) died during follow-up and 42 $(21.6 \%)$ within 1 year from surgery. One-year mortality was significantly associated with age, tumor location, NLR, RDW, LDH and FIB.

\section{Univariate and Multivariate Analyses}

In univariate Cox analysis, 7 predictive variables were associated with higher risk of early death within 1 year after GTR: older age $(P=0.013)$, occurrence of non-seizures symptoms $(P=0.042)$, special tumor positions $(\mathrm{P}=0.046)$, higher NLR $(P=0.015)$, higher RDW $(P=0.019)$, higher LDH $(P=0.005)$, and higher FIB $(P=0.044)$ (Figure 1A). The predictive performance of all significant characteristics was assessed using the AUCs obtained from ROC curves. According to the AUCs, LDH was the leading factor for predicting early death, which showed its significant predictive potential ( 0.625 vs 0.565 to 0.615 ; Figure 2 ). In multivariate Cox analysis, tumor location $(\mathrm{HR}=1.590,95 \% \mathrm{CI}$ 1.107-2.284; $\mathrm{P}=0.012)$, NLR $(\mathrm{HR}=3.164,95 \%$ CI $1.107-$ 9.041; $\mathrm{P}=0.032)$ and $\mathrm{LDH}(\mathrm{HR}=2.580,95 \% \mathrm{CI} 2.875-$ $5.528 ; \mathrm{P}=0.002$ ) remained independently associated with 1 year mortality after GTR (Figure 1B).

\section{Survival Analysis of Independent Predictive Hematological Markers}

As shown in Figure 3A, GBM patients with NLR $>1.706$ showed a significantly poorer OS than that of GBM patients with NLR $\leq 1.706(\mathrm{P}=0.009)$. Similarly, the OS of patients in the LDH high-risk group was significantly lower than that of patients in the LDH low-risk group ( $\mathrm{P}=0.004$, Figure $3 \mathrm{~B}$ ). The results indicated that
Table 2 Comparison of Preoperative Risk Factors for Postoperative Death Within I Year After GTR

\begin{tabular}{|c|c|c|c|}
\hline Characteristics & $\begin{array}{l}\text { Death } \\
\text { Within I } \\
\text { Year (42) }\end{array}$ & $\begin{array}{l}\text { Death After } \\
\text { I Year (I52) }\end{array}$ & P-value \\
\hline $\begin{array}{l}\text { Age, years } \\
\text { Median } \\
\text { Range }\end{array}$ & $\begin{array}{l}56.5 \\
21-80\end{array}$ & $\begin{array}{l}55 \\
19-78\end{array}$ & 0.022 \\
\hline $\begin{array}{l}\text { Clinical history, } \\
\text { days } \\
\text { Median } \\
\text { Range }\end{array}$ & $\begin{array}{l}20 \\
2-365\end{array}$ & $\begin{array}{l}20 \\
I-365\end{array}$ & 0.452 \\
\hline $\begin{array}{l}\text { Sex, } \mathbf{n}(\%) \\
\quad \text { Male } \\
\text { Female }\end{array}$ & $\begin{array}{l}25(59.5) \\
17(40.5)\end{array}$ & $\begin{array}{l}90(59.2) \\
62(40.8)\end{array}$ & 0.971 \\
\hline $\begin{array}{l}\text { Size, cm, n (\%) } \\
\quad \leq 5 \\
>5\end{array}$ & $\begin{array}{l}29(69.0) \\
13(31.0)\end{array}$ & $\begin{array}{l}94(61.8) \\
58(38.2)\end{array}$ & 0.391 \\
\hline $\begin{array}{l}\text { First presenting } \\
\text { symptoms, n (\%) } \\
\text { Seizures } \\
\text { Others }\end{array}$ & $\begin{array}{l}2(9.1) \\
40(95.2)\end{array}$ & $\begin{array}{l}20(13.2) \\
132(86.8)\end{array}$ & 0.213 \\
\hline $\begin{array}{l}\text { Tumor location, } \\
\text { n (\%) } \\
\text { Frontal } \\
\text { Temporal } \\
\text { Mixed } \\
\text { Others }\end{array}$ & $\begin{array}{l}5(11.9) \\
3(7.1) \\
29(69.0) \\
5(11.9)\end{array}$ & $\begin{array}{l}40(26.3) \\
40(26.3) \\
40(26.3) \\
32(21.1)\end{array}$ & $<0.001$ \\
\hline $\begin{array}{l}\text { NLR, n (\%) } \\
\quad \leq 1.706 \\
>1.706\end{array}$ & $\begin{array}{l}4(9.5) \\
38(90.5)\end{array}$ & $\begin{array}{l}46(30.3) \\
106(69.7)\end{array}$ & 0.012 \\
\hline $\begin{array}{l}\text { PLR, n (\%) } \\
\quad \leq 87.857 \\
>87.857\end{array}$ & $\begin{array}{l}4(9.5) \\
38(90.5)\end{array}$ & $\begin{array}{l}36(23.7) \\
116(76.3)\end{array}$ & 0.073 \\
\hline $\begin{array}{l}\text { LMR, n (\%) } \\
\quad \leq 3.067 \\
>3.067\end{array}$ & $\begin{array}{l}I I(26.2) \\
3 I(73.8)\end{array}$ & $\begin{array}{l}56(36.8) \\
96(63.2)\end{array}$ & 0.199 \\
\hline $\begin{array}{l}\text { PNI, n (\%) } \\
\quad \leq 56.5 \\
>56.5\end{array}$ & $\begin{array}{l}35(83.3) \\
7(16.7)\end{array}$ & $\begin{array}{l}\mid 34(88.2) \\
\mid 8(\mid 1.8)\end{array}$ & 0.409 \\
\hline $\begin{array}{l}\text { AGR, } \mathbf{n}(\%) \\
\quad \leq 1.908 \\
>1.908\end{array}$ & $\begin{array}{l}14(33.3) \\
28(66.7)\end{array}$ & $\begin{array}{l}47(30.9) \\
105(69.1)\end{array}$ & 0.766 \\
\hline $\begin{array}{l}\text { RDW, \%, n (\%) } \\
\leq 12.8 \\
>12.8\end{array}$ & $\begin{array}{l}5(11.9) \\
37(88.1)\end{array}$ & $\begin{array}{l}47(30.9) \\
105(69.1)\end{array}$ & 0.014 \\
\hline
\end{tabular}

(Continued) 
Table 2 (Continued).

\begin{tabular}{|c|c|c|c|}
\hline Characteristics & $\begin{array}{l}\text { Death } \\
\text { Within I } \\
\text { Year (42) }\end{array}$ & $\begin{array}{l}\text { Death After } \\
\text { I Year (I52) }\end{array}$ & P-value \\
\hline \multicolumn{4}{|l|}{$\begin{array}{l}\text { GLC, mmol/L, } \\
\text { n (\%) }\end{array}$} \\
\hline$\leq 6.57$ & $32(76.2)$ & 134(88.2) & 0.051 \\
\hline$>6.57$ & $10(23.8)$ & $18(11.8)$ & \\
\hline \multicolumn{4}{|l|}{ LDH, U/L, n (\%) } \\
\hline$\leq 182$ & $16(38.1)$ & $96(63.2)$ & 0.004 \\
\hline$>182$ & $26(63.2)$ & $56(36.8)$ & \\
\hline \multicolumn{4}{|l|}{ FIB, g/L, n (\%) } \\
\hline$\leq 3.81$ & $3 I(73.8)$ & $132(86.8)$ & 0.041 \\
\hline$>3.81$ & $\mathrm{II}(26.2)$ & $20(13.2)$ & \\
\hline \multicolumn{4}{|l|}{ DD, mg/L, n (\%) } \\
\hline$\leq 0.183$ & $28(66.7)$ & $122(80.3)$ & 0.063 \\
\hline$>0.183$ & $14(33.3)$ & $30(19.7)$ & \\
\hline
\end{tabular}

Abbreviations: NLR, the neutrophil-to-lymphocyte ratio; PLR, the platelet-tolymphocyte ratio; LMR, the lymphocyte -to- monocyte ratio; PNI, the prognostic nutrition index; AGR, albumin-to-globulin ratio; RDW, red blood cell distribution width; GLC, glucose; LDH, lactate dehydrogenase; FIB, fibrinogen; DD, D-dimer.

preoperative NLR and LDH level may be used in clinical practice to identify high-risk patients.

\section{Development of the Nomogram Related to Early Death}

The prognostic nomogram that integrated all significant factors for early death in multivariate Cox regression analysis is shown in Figure 4A. Every covariate was allocated a score based on the multivariate Cox analysis, and the nomogram score termed total points was generated by summing risk points scores of all the covariates. Tumor location had maximum score points (ranging from 25 to 100) compared to other clinical factors. Among them, mixed lobe location had higher risk scores for early mortality compared with patients with tumor located in other positions. The C-index of the nomogram for early mortality prediction was 0.795 . The calibration plot for the probability of survival at 1 year after GTR showed an optimal agreement between the prediction by nomogram and actual observation (Figure 4B). The results of DCA showed that the nomogram could yield clinical net benefits (Figure 4C).

\section{Discussion}

In recent decades, despite continuous improvements in neurosurgery techniques and innovations in new treatment methods, such as immunotherapy and molecular-targeted therapy,
A
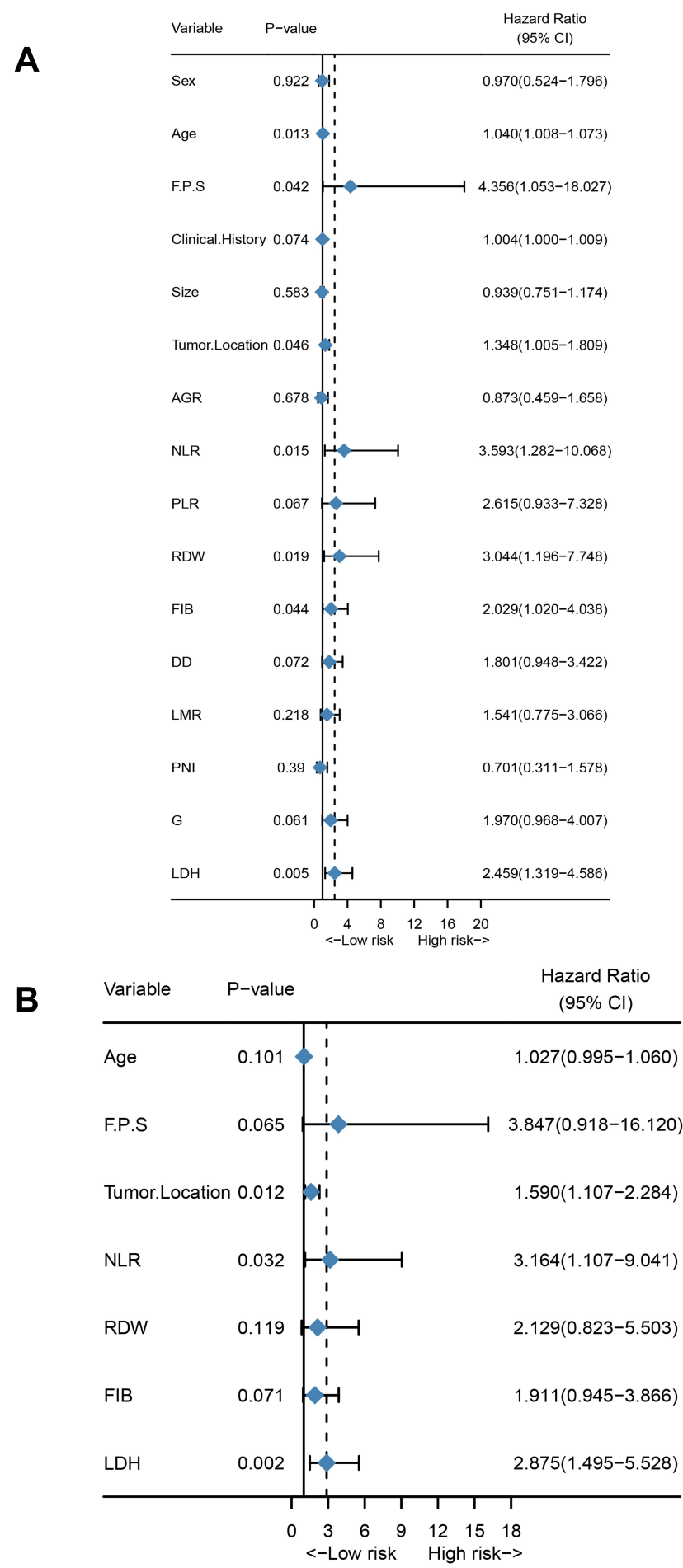

Figure I Preoperative predictive factors for I-year mortality after GTR according to COX regression.

Notes: (A) Forest plot of univariate Cox regression analysis of all clinical covariates; (B) Forest plot of multivariate Cox regression analysis of significant clinical covariates.

Abbreviations: GTR, gross total resection; FPS, first presenting symptoms.

the survival time of patients with GBM is still not optimistic. ${ }^{2,29,30}$ It is noteworthy that a certain number of GBM patients with good preoperative and postoperative 


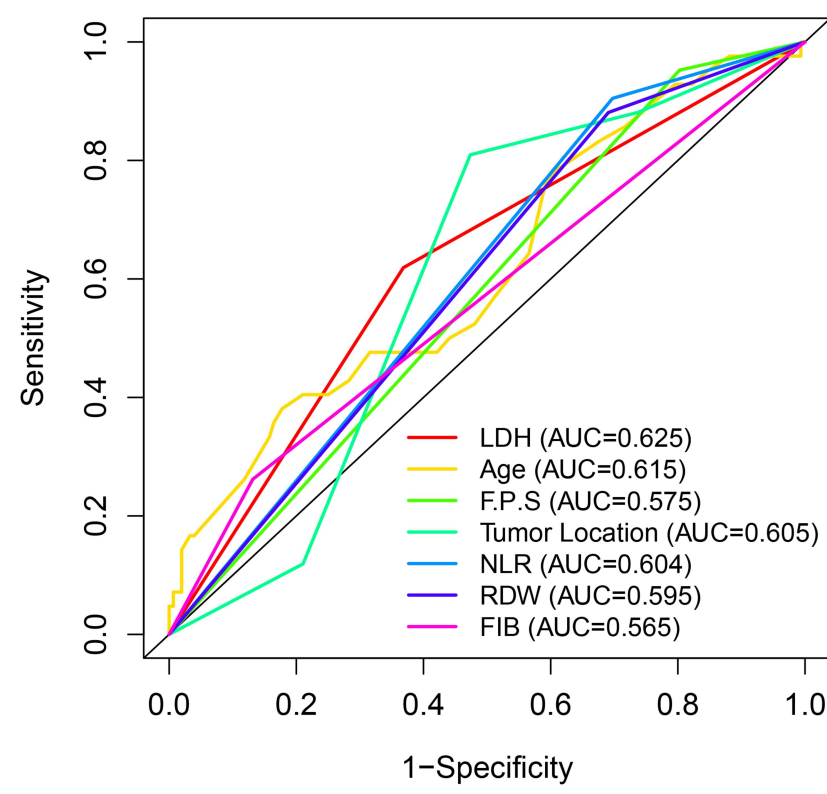

Figure 2 Receiver operating characteristic (ROC) curves of significant clinical covariates for overall survival.

Abbreviation: FPS, first presenting symptoms.

health status, who received standard treatment protocols, should have better clinical outcomes, but instead had an endpoint event ie, death within 1 year. Thus, we focused on IDH-wt GBM patients with preoperative and postoperative KPS scores $\geq 70$ (ECOG PS scores $\geq 1$ ) and receiving standard treatment including GTR, followed by concurrent chemoradiotherapy and adjuvant temozolomide. In addition, accumulating studies have demonstrated that peripheral blood test parameters play a remarkable role in the prognosis of various malignant tumors, such as lung cancer, gastric cancer, and soft tissue sarcoma, as well as GBM. ${ }^{31-33}$ Unlike genetic biomarkers, preoperative hematological markers, which are detected via noninvasive procedures and are more cost-effective, can be easily obtained from routine blood tests. In the present study, we included 194 homogenous IDH-wt GBM patients to assess preoperative risk factors for early death. Further, we collected all hematological indicators of these patients that have been proven to be prognostic in previous studies, and incorporated them into subsequent analyses as binary variables based on the ROC curve. Multivariable analyses identified the following three factors as predictive of early death: tumor location, preoperative NLR and serum LDH level. To further improve the accuracy of the prediction of early mortality, we established a nomogram based on clinical and hematological parameters. The $\mathrm{C}$-index of the nomogram was 0.795 , which showed excellent predictive power. This may guide the
A

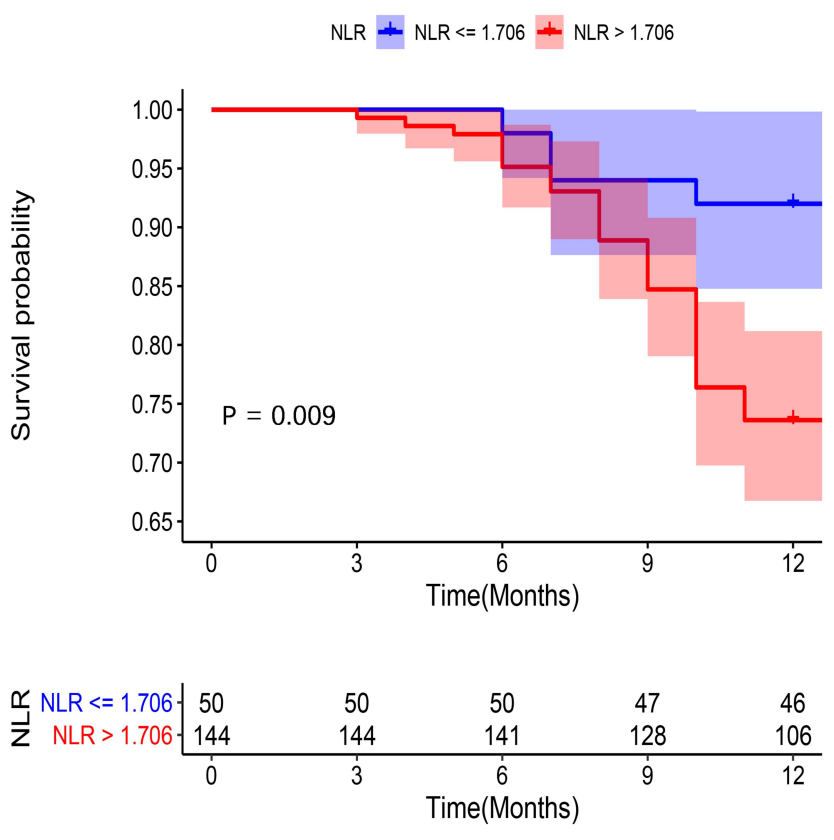

B

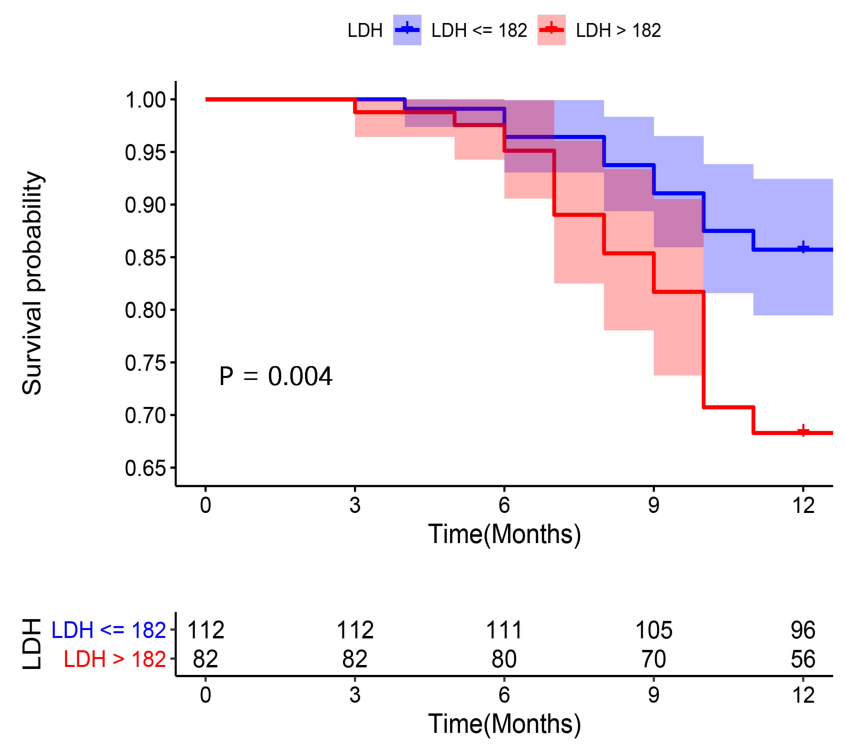

Figure 3 Comparison of survival curves of GBM patients at different hematological groups.

Notes: (A) Kaplan-Meier curves of overall survival for patients in groups stratified by NLR; (B) Kaplan-Meier curves of overall survival for patients in groups stratified by LDH.

neurosurgeons in early identification of GBM patients with a high probability of early mortality before surgery, so as to instruct these high-risk patients to review head MRI more frequently and regularly, and to increase follow-up of these patients.

In the present study, tumor location had a significant effect on the occurrence of early death. GBM patients with 
A

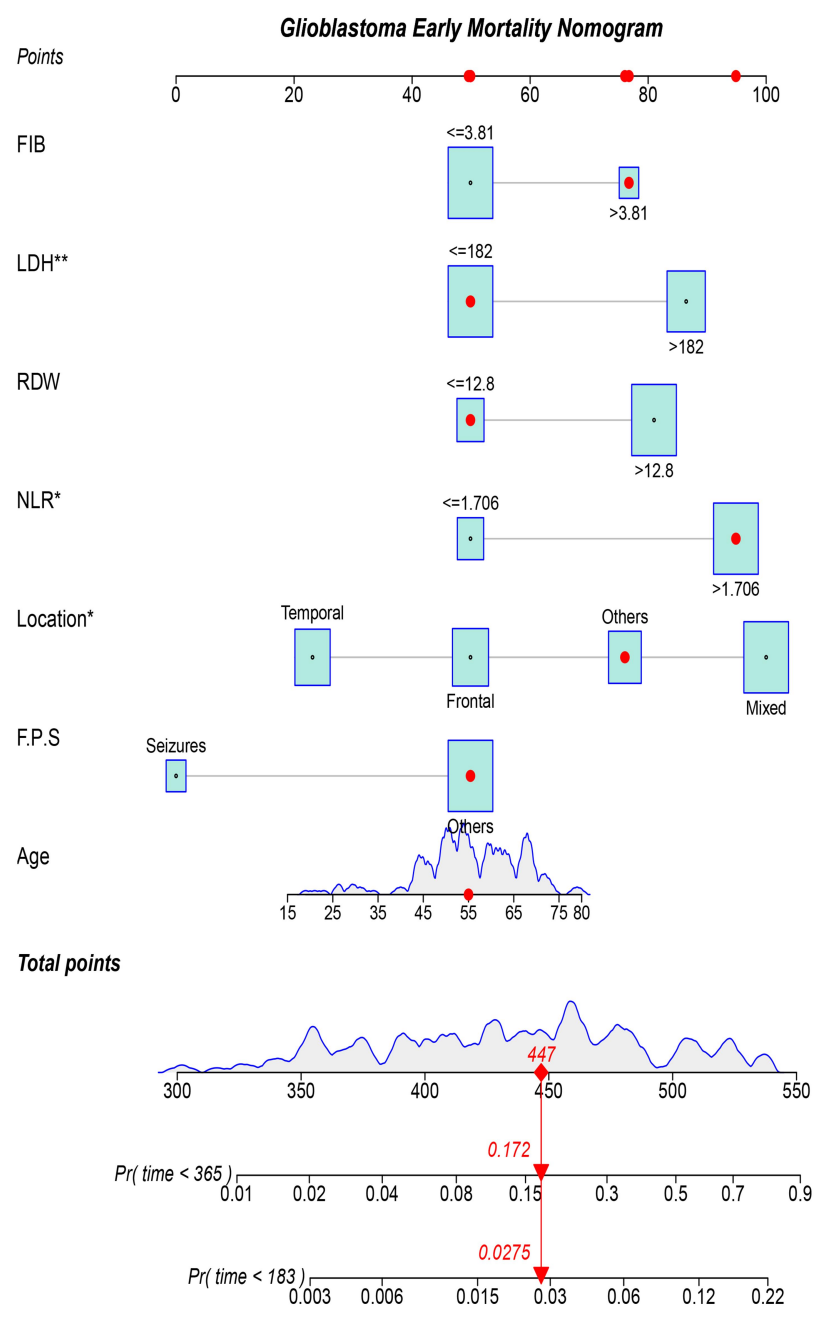

B

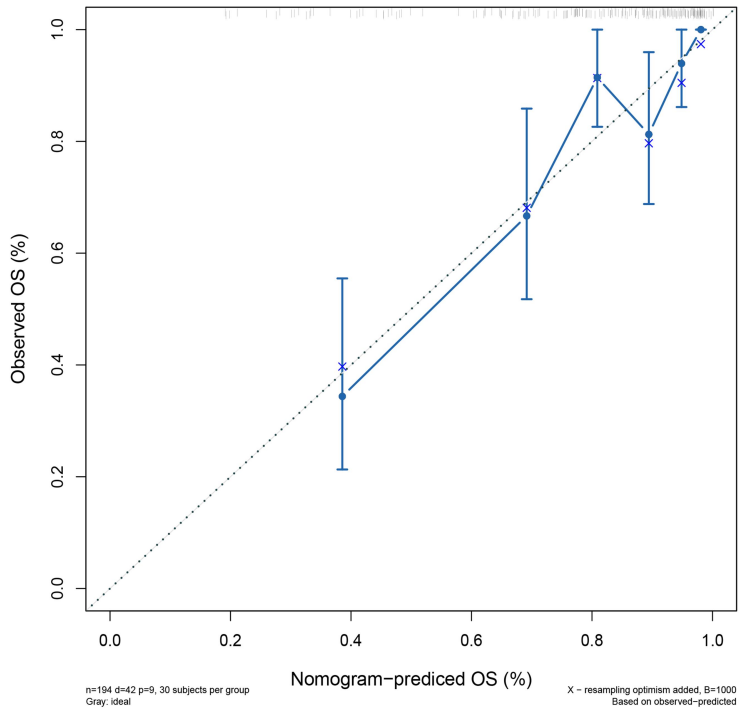

C

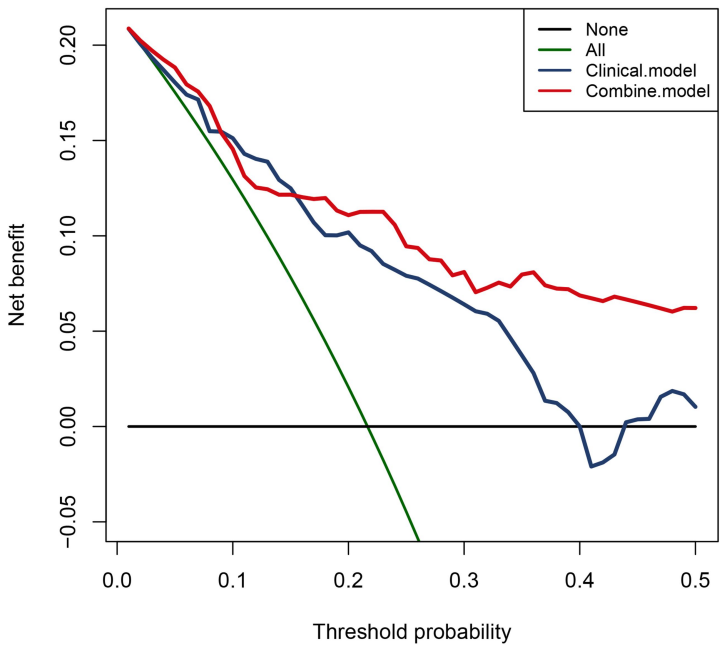

Figure 4 Establish an overall survival nomogram to predict the probability of early death in GBM.

Notes: (A) Nomogram to predict the probability of patient mortality based on significant factors; (B) Calibration chart to verify the accuracy of the nomogram. Nomogram performance is shown by the plot, relative to the 30-degree line, which represents the ideal prediction; (C) Decision curve analysis of the nomogram. Clinical model: only preoperative clinical parameters; Combine model: preoperative clinical parameters + hematological parameters.

Abbreviation: FPS, first presenting symptoms.

tumors located in mixed areas had higher risk for early mortality than those with tumors located in the frontal and temporal lobes. In addition, tumor location was the dominant factor compared to other clinical factors in the nomogram. The prognostic role of tumor location for patients with GBM has been evaluated by previous investigations. ${ }^{34} \mathrm{~A}$ recent publication by Dietterle et al revealed that the hazard ratio of unilobar tumors is twice that of multilobar tumors. ${ }^{35}$ Tumors located in mixed areas are adjacent to many critical arteriovenous structures and functional areas. Therefore, tumors located in these regions may increase the difficulty of operative and incidence of complications, which could result in early mortality and unfavorable long-term clinical outcomes.

The effect of NLR on early mortality was also apparent in our study results. We demonstrated that patients with high-NLR have worse OS compared with low-NLR patients undergoing GTR. Recently, there has also been considerable evidence that inflammation is closely related to the occurrence and development of malignant tumors, the concept of cancer-related inflammation has also been proposed. ${ }^{12}$ Some inflammatory parameters are associated 
with adverse prognostic outcomes of gliomas, the most representative of which is NLR. ${ }^{36}$ Published qualitative results have shown that neutrophil can promote the progression of glioma by remodeling the extracellular matrix and promoting angiogenesis, and that certain subsets of T-lymphocytes can instead inhibit it via induction of cytotoxic cell death and cytokine production. ${ }^{12,37}$ Accordingly, high neutrophil and low T-cell (elevated NLR) in GBM was correlated with poorer outcome. ${ }^{15,38}$ However, the predictive value of NLR for early death has not yet been reported in GBM patients receiving standard treatment. We believe that preoperative NLR might represent cancerrelated systemic inflammation response to evaluate the early mortality of GBM.

In our study, we also further observed that preoperative serum LDH level is significantly associated with 1-year mortality. LDH is released from cells upon damage to their cytoplasmic membrane, and is both a metabolic and an immune surveillance prognostic biomarker. Increased LDH level is a negative prognostic biomarker, not only because it is a key enzyme involved in tumor metabolism, but also because it allows cancer cells to suppress and evade the immune system by altering the tumor microenvironment. ${ }^{39-41}$ To the best of our knowledge, the relationship between preoperative LDH level and early mortality in GBM patients has not yet been investigated. The present findings suggested that serum LDH level might serve as a preoperative predictive factor to identify early death of GBM patients.

However, some limitations of the present study should be considered. First, this was a single-center retrospective study that may have led to bias in selection and analysis. Second, since the values of the hematological markers were entered into Cox regression as categorical variables, the optimal cutoff value needs to be further verified in future studies. Finally, the number of patients is somewhat small to make a final conclusion on this matter. Considering the limited number of the study conducted, further studies are needed to identify and characterize of the GBM patients with a high probability of early mortality.

\section{Conclusion}

In summary, tumor location, NLR, and serum LDH level are independent predictors for 1-year mortality after standard treatment. To the best of our knowledge, this is the first study to investigate the predictive value of preoperative NLR and serum LDH level for early death in GBM patients with standard therapy. Following the results, increased preoperative LDH or NLR should lead to more frequent and regular head MRI reviews to monitor tumor progression.

\section{Ethics Approval}

This study was approved by the Ethics Committee of the First Affiliated Hospital of Zhengzhou University.

\section{Acknowledgments}

Chao Zhao and Longqing Li are co-first authors for this study.

\section{Author Contributions}

All authors contributed to data analysis, drafting or revising the article, have agreed on the journal to which the article will be submitted, gave final approval of the version to be published, and agree to be accountable for all aspects of the work.

\section{Funding}

No funding was received for this research.

\section{Disclosure}

The authors report no conflicts of interest in this work.

\section{References}

1. Ostrom Q, Gittleman H, Truitt G, Boscia A, Kruchko C, BarnholtzSloan J. CBTRUS statistical report: primary brain and other central nervous system tumors diagnosed in the United States in 2011-2015. Neuro-Oncology. 2018;20:iv1-iv86. doi:10.1093/neuonc/noy131

2. Tan A, Ashley D, López G, Malinzak M, Friedman H, Khasraw M. Management of glioblastoma: state of the art and future directions. $C A$ Cancer J Clin. 2020;70:299-312. doi:10.3322/caac.21613

3. Taphoorn M, Sizoo E, Bottomley A. Review on quality of life issues in patients with primary brain tumors. Oncologist. 2010;15(6):618-626. doi:10.1634/theoncologist.2009-0291

4. Louis D, Perry A, Reifenberger G, et al. The 2016 world health organization classification of tumors of the central nervous system: a summary. Acta Neuropathol. 2016;131(6):803-820. doi:10.1007/ s00401-016-1545-1

5. Stupp R, Hegi M, Mason W, et al. Effects of radiotherapy with concomitant and adjuvant temozolomide versus radiotherapy alone on survival in glioblastoma in a randomised Phase III study: 5-year analysis of the EORTC-NCIC trial. Lancet Oncol. 2009;10 (5):459-466. doi:10.1016/S1470-2045(09)70025-7

6. Stupp R, Mason W, van den Bent M, et al. Radiotherapy plus concomitant and adjuvant temozolomide for glioblastoma. $N$ Engl J Med. 2005;352(10):987-996. doi:10.1056/NEJMoa043330

7. Koshy M, Villano J, Dolecek T, et al. Improved survival time trends for glioblastoma using the SEER 17 population-based registries. J Neurooncol. 2012;107(1):207-212. doi:10.1007/s11060-011-0738-7

8. Ostrom Q, Cote D, Ascha M, Kruchko C, Barnholtz-Sloan J. Adult glioma incidence and survival by race or ethnicity in the United States from 2000 to 2014. JAMA oncol. 2018;4(9):1254-1262. doi:10.1001/ jamaoncol.2018.1789 
9. Lacroix M, Abi-Said D, Fourney D, et al. A multivariate analysis of 416 patients with glioblastoma multiforme: prognosis, extent of resection, and survival. J Neurosurg. 2001;95(2):190-198. doi:10.31 71/jns.2001.95.2.0190

10. Lamborn K, Chang S, Prados M. Prognostic factors for survival of patients with glioblastoma: recursive partitioning analysis. Neuro Oncology. 2004;6(3):227-235. doi:10.1215/S1152851703000620

11. Jeremic B, Milicic B, Grujicic D, Dagovic A, Aleksandrovic J. Multivariate analysis of clinical prognostic factors in patients with glioblastoma multiforme treated with a combined modality approach. J Cancer Research Clin Oncol. 2003;129(8):477-484. doi:10.1007/ s00432-003-0471-5

12. Mantovani A, Allavena P, Sica A, Balkwill F. Cancer-related inflammation. Nature. 2008;454(7203):436-444. doi:10.1038/nature 07205

13. McMillan D. The systemic inflammation-based glasgow prognostic score: a decade of experience in patients with cancer. Cancer Treat Rev. 2013;39(5):534-540. doi:10.1016/j.ctrv.2012.08.003

14. Bao Y, Yang M, Jin C, et al. Preoperative hematologic inflammatory markers as prognostic factors in patients with glioma. World Neurosurg. 2018;119:e710-e716. doi:10.1016/j.wneu.2018.07.252

15. Wang P, Meng Z, Song H, et al. Preoperative changes in hematological markers and predictors of glioma grade and survival. Front Pharmacol. 2018;9:886. doi:10.3389/fphar.2018.00886

16. Mandoj C, Tomao L, Conti L.Coagulation in brain tumors: biological basis and clinical implications. Front Neurol. 2019;10(181). doi:10.3389/fneur.2019.00181

17. SE N, Guarnaccia L, Locatelli M, et al. Significance and prognostic value of the coagulation profile in patients with glioblastoma: implications for personalized therapy. World Neurosurg. 2019;121:e621e629. doi:10.1016/j.wneu.2018.09.177

18. Hagan K, Bhavsar S, Arunkumar R, et al. Association between perioperative hyperglycemia and survival in patients with glioblastoma. J Neurosurg Anesthesiol. 2017;29(1):21-29. doi:10.10 97/ANA.0000000000000339

19. Xu W, Li F, Xu Z, et al. Preoperative albumin-to-globulin ratio and prognostic nutrition index predict prognosis for glioblastoma. Onco Targets Ther. 2017;10:725-733. doi:10.2147/OTT.S127441

20. Zhou X, Dong H, Yang Y, et al. Significance of the prognostic nutritional index in patients with glioblastoma: A retrospective study. Clin Neurol Neurosurg. 2016;151:86-91. doi:10.1016/j. clineuro.2016.10.014

21. Kim J, Han J, Jang Y. High-capacity glycolytic and mitochondrial oxidative metabolisms mediate the growth ability of glioblastoma. Int J Oncol. 2015;47(3):1009-1016. doi:10.3892/ijo.2015.3101

22. RF L, Li M, Yang Y, Mao Q, Liu Y-H. Significance of pretreatment red blood cell distribution width in patients with newly diagnosed glioblastoma. Med Sci Monitor. 2017;23:3217-3223. doi:10.12659/ MSM.905204

23. Bouchart C, Trépant A, Hein M, Van Gestel D, Demetter P. Prognostic impact of glioblastoma stem cell markers OLIG2 and CCND2. Cancer Med. 2020;9(3):1069-1078. doi:10.1002/cam4.2592

24. Wasserman J, Nicholas G, Yaworski R, et al. Radiological and pathological features associated with IDH1-R132H mutation status and early mortality in newly diagnosed anaplastic astrocytic tumours. PLoS One. 2015;10(4):e0123890. doi:10.1371/journal.pone.0123890

25. Karnofsky D, Burchenal J. The clinical evaluation of chemotherapeutic agents in cancer. In: MacLeod C, editor. Evaluation of Chemotherapeutic Agents. New York, NY: Columbia University Press; 1949:191-205.
26. Pirracchio R, Resche-Rigon M, Bresson D, et al. One-year outcome after neurosurgery for intracranial tumor in elderly patients. $J$ Neurosurg Anesthesiol. 2010;22(4):342-346. doi:10.1097/ANA.0b 013e3181e6daa2

27. Villani V, Pace A, Vidiri A, et al. Phase II study of weekly carboplatin in pretreated adult malignant gliomas. J Neurooncol. 2019;144 (1):211-216

28. Oken M, Creech R, Tormey D, et al. Toxicity and response criteria of the eastern cooperative oncology group. Am J Clin Oncol. 1982;5 (6):649-655. doi:10.1097/00000421-198212000-00014

29. Lynes J, Nwankwo A, Sur H, et al. Biomarkers for immunotherapy for treatment of glioblastoma. J Immunotherapy Cancer. 2020;8:1. doi:10.1136/jitc-2019-000348

30. Le Rhun E, Preusser M, Roth P, et al. Molecular targeted therapy of glioblastoma. Cancer Treat Rev. 2019;80:101896. doi:10.1016/j. ctrv.2019.101896

31. Charrier M, Mezquita L, Lueza B, et al. Circulating innate immune markers and outcomes in treatment-naïve advanced non-small cell lung cancer patients. Eur J Cancer. 2019;108:88-96. doi:10.1016/j. ejca.2018.12.017

32. $\mathrm{Xu} \mathrm{Z}, \mathrm{Xu} \mathrm{W}$, Cheng $\mathrm{H}$, et al. The prognostic role of the platelet-lymphocytes ratio in gastric cancer: a meta-analysis. PLoS One. 2016;11(9):e0163719. doi:10.1371/journal.pone.0163719

33. Li L, Bai Z, Zhang L, et al. Meta-analysis of hematological biomarkers as reliable indicators of soft tissue sarcoma prognosis. Front Oncol. 2020;10:30. doi:10.3389/fonc.2020.00030

34. Adeberg S, Bostel T, König L, Welzel T, Debus J, Combs S. A comparison of long-term survivors and short-term survivors with glioblastoma, subventricular zone involvement: a predictive factor for survival? Radiation Oncol. 2014;9:95. doi:10.1186/1748-717X-9-95

35. Dietterle J, Wende T, Wilhelmy F, et al. The prognostic value of peri-operative neurological performance in glioblastoma patients. Acta Neurochir. 2020;162(2):417-425. doi:10.1007/s00701-01904136-4

36. Wang D, Kang K, Lin Q, Hai J. Prognostic significance of preoperative systemic cellular inflammatory markers in gliomas: a systematic review and meta-analysis. Clin Transl Sci. 2020;13(1):179-188. doi:10.1111/cts. 12700

37. Mostofa A, Punganuru S, Madala H, Al-Obaide M, Srivenugopal K. The process and regulatory components of inflammation in brain oncogenesis. Biomolecules. 2017;7(4):2. doi:10.3390/biom7020034

38. Han S, Liu Y, Li Q, Li Z, Hou H, Wu A. Pre-treatment neutrophil-tolymphocyte ratio is associated with neutrophil and T-cell infiltration and predicts clinical outcome in patients with glioblastoma. $B M C$ Cancer. 2015;15:617. doi:10.1186/s12885-015-1629-7

39. Ding J, Karp J, Emadi A. Elevated lactate dehydrogenase (LDH) can be a marker of immune suppression in cancer: interplay between hematologic and solid neoplastic clones and their microenvironments. Cancer Biomarkers. 2017;19(4):353-363. doi:10.3233/CBM-160336

40. Petrelli F, Cabiddu M, Coinu A, et al. Prognostic role of lactate dehydrogenase in solid tumors: a systematic review and meta-analysis of 76 studies. Acta Oncologica. 2015;54(7):961-970. doi:10.3109/0284186X.2015.1043026

41. Zhang J, Yao Y, Li B, Yang Q, Zhang P, Wang H. Prognostic value of pretreatment serum lactate dehydrogenase level in patients with solid tumors: a systematic review and meta-analysis. Sci Rep. 2015;5:9800. doi:10.1038/srep09800 


\section{Publish your work in this journal}

Cancer Management and Research is an international, peer-reviewed open access journal focusing on cancer research and the optimal use of preventative and integrated treatment interventions to achieve improved outcomes, enhanced survival and quality of life for the cancer patient.

The manuscript management system is completely online and includes a very quick and fair peer-review system, which is all easy to use. Visit http://www.dovepress.com/testimonials.php to read real quotes from published authors.

Submit your manuscript here: https://www.dovepress.com/cancer-management-and-research-journal 